radiologistas, a problemática diagóstica da TEP parece encerrada. Assim seja.

Terra-Filho et al $^{(7)}$ apresentam um relato de caso que reflete a grande experiência de seu grupo no manejo cirúrgico da TEP crônica, isto é, da hipertensão pulmonar tromboembólica crônica. Sua casuística e resultados obtidos são de padrão internacional.

Maciel et $a l^{(8)}$ apresentam uma revisão igualmente concisa sobre profilaxia da TEV em pacientes submetidos a procedimentos vídeolaparoscópicos, a propósito de um caso acompanhado. Avanços nos procedimento cirúrgicos, fazendo-os menos invasivos, menos cruentos e mais ágeis, não os tornam isentos de riscos de TEV, merecendo avaliação e tromboprofilaxia correspondente.

A circulação pulmonar é uma realidade entre nós.

SÉRgIO SALDANHA MENNA BARRETO (TE SBPT)

Prof. Titular da Faculdade de Medicina da Universidade Federal do Rio Grande do Sul

\section{REFERÊNCIAS}

1. Moser KM. Pulmonary embolism. Am Rev Respir Dis1977; 115:829-51.

2. Dalen JE, Alpert JS. Natural history of pulmonary embolism. Prog Cardiovasc Dis.1975; 17:259-70

3. Moser MK. Venous thromboembolism. Am Rev Respir Dis 1990; 141:235-49.

4. Yoo HHB, Mendes FG, Alem CER. Achados clinicopatológicos na tromboembolia pulmonar: estudos de 24 anos de autopsias. J Bras Pneumol 2002: 30:426-32.

5. Silva IS, Müller NL. Diagnóstico por imagens do tromboembolismo pulmonar agudo. J Bras Pneumol 2004; 30: 474-9.

6. Remy-Jardim M, Remy J, Wattime L et al. Central pulmonary thromboembolism: diagnosis with spiral volumetric CT with single-breatjh technique comparison with pulmonary angiography. Radiology 1992, 185:381-7.

7. Terra-Filho M, Ribeiro SCC, Souza R, Jatene FB. Tromboendarterectomia pulmonar em paciente com 80 anos de idade. J Bras Pneumol 2004; 30:485-7.

8. Maciel R, Menna Barreto SS. Tromboprofilaxia na colecistectomia videolaparoscópica. J Bras Pneumol 2004; 30:480-4.

\title{
0 Jornal Brasileiro de Pneumologia e a Internet
}

O Jornal Brasileiro de Pneumologia (JBP) manteve contínuo processo de aprimoramento ao longo de seus 30 anos. As mudanças recentes estiveram intimamente ligadas a Internet. A idexação do JBP na base de dados SciELO ${ }^{(1)}$ permitiu o acesso gratuito e integral do conteúdo do JBP não só em português, mas também em inglês (www.jornaldepneumologia.com.br ou alternativamente www.scielo.br/jbpneu). Atualmente Editores Associados no Brasil e exterior, participam diretamente de todo o processo de revisão dos artigos, desde a sugestão de revisores até a decisão final. Esse processo seria inimaginável sem o auxílio da Internet. $\mathrm{Na}$ outra ponta, o número de artigos submetidos ao JBP saltou de 77 em 2002 para $134 \mathrm{em}$ 2003. Apoiado nas facilidades de comunicação, aumentamos em paralelo o número de revisores ad hoc, que saltou de $53 \mathrm{em} 2002$ para $130 \mathrm{em}$ 2003. Com isso conseguimos que o tempo médio da primeira revisão caisse de 44 + 32 para $29{ }^{ \pm} 16$ dias $^{(2)}$. A agilidade veio sem perda da qualidade das revisões, o índice de rejeição de artigos tem na realidade aumentado.
Nesse momento estamos na fase final de implementação de programa que facilitará a submissão e revisão dos artigos, que serão feitos integralmente na Internet. Convidamos todos os membros da sociedade, incluindo autores, revisores e pesquisadores que tenham interesse em contribuir com o JBP, a preencher o seu cadastro via internet. Uma vez conectado, você não perderá mais do que alguns minutos. 0 cadastro é simples, e será essencial para atualizarmos o nosso banco de dados. Acesse: www.jornaldepneumologia.com.br/sgp Preencha o seu cadastro agora

$$
\begin{aligned}
& \text { GERALDO LORENZI-FILHO } \\
& \text { EDITE SBPT) } \\
& \text { EITOR }
\end{aligned}
$$

\section{REFERÊNCIAS}

1. Queluz, THAT. Admissão do Jornal de Pneumologia na SciElO Brasil: uma vittória com novos desafios. J Pneumol 2002;28:1X-X

2. Lorenzi-Filho, G. 0 espírito do Jornal de Pneumologia. J Pneumol 2003;29:335. 